\title{
Design of an Impulse Radiating Antenna Using a Curved TEM-Wire Fed Parabola
}

\author{
Donghyun Lee, ${ }^{1}$ Haengseon Lee, ${ }^{1}$ Sunmook Hwang, ${ }^{2}$ and Jaewoon Ahn ${ }^{2}$ \\ ${ }^{1}$ Department of Electronic Engineering, Sogang University, 1 Sinsu-dong, Mapo-gu, Seoul, Republic of Korea \\ ${ }^{2}$ R\&D Department 2, Hanwha Corporation Gumi Plant, 258 Gongdan-dong, Gumi-si, \\ Gyungsangbuk-do, Republic of Korea \\ Correspondence should be addressed to Haengseon Lee, leehs95@sogang.ac.kr \\ Received 18 April 2012; Accepted 25 June 2012 \\ Academic Editor: Hon Tat Hui
}

Copyright () 2012 Donghyun Lee et al. This is an open access article distributed under the Creative Commons Attribution License, which permits unrestricted use, distribution, and reproduction in any medium, provided the original work is properly cited.

A design procedure for a TEM-wire fed parabolic antenna is given for impulse radiation, which is suitable for low cost fabrication. A simple wire-type TEM horn and a parabolic reflector are used to achieve ultra-wide bandwidth and high directivity at the same time. Equations for parametric curves of a TEM-wire horn are presented and are used to investigate the relation among their shapes, bandwidth, and directivity. It is also found that wire separation near the focal point limits the high frequency directivity.

\section{Introduction}

Very wide band antennas have been given much interest from commercial and military applications such as communication services, electromagnetic compatibility, radar technologies, and electronic warfare [1]. To accommodate very high speed wireless data demand, ultra-wide communication services are proposed and UWB antennas have been hot research topics [2]. Very wide band antennas are utilized to test immunity to electrostatic discharges or wide band interferences. Applications for radars and electronic warfare also need very wide band antennas to radiate or receive short pulse energy into or from the air. In most of the researches, ultra-wide band antennas achieve bandwidth requirement using smooth transition from the feed to radiating structures like exponential, polynomial, or linear tapers $[3,4]$. At the same time, high directivity has been attained by adopting array structures or utilizing reflectors [5-8]. To attain both requirements, the size and weight of those antennas are very large and heavy and tapering schemes depend on the particular structure of the tapered feed horn antenna, which deserves careful studies [9-12].

In this paper, an ultra-wide band antenna with high gain is proposed, which is composed of a TEM-wire-type feed horn and a parabolic reflector. Compared with other
TEM horn reflector antennas [7-10], the antenna structure is suitable for low cost fabrication in that TEM-wire horns can be built by bending copper wires or pipes, and inexpensive parabolas for satellite broadcasting reception can be utilized without any additional components such as balun or power divider. The bandwidth and directivity needed are met through adjusting parameters of the wire-type feed horn structure of a proposed parametric curve equation. Various feed horn tapering schemes by adjusting parameters of the proposed equations as applied to parabolic reflectors are examined through simulations and measurements. The directivities and return losses of combined antenna using TEM-wire feed horn are closely related to each other, and compromise is needed to satisfy design requirement.

In the following sections, the whole antenna structure is divided into a coaxial cable to TEM-wire horn transition, tapered feed arms, and a reflector. Design considerations of each component and restrictions are outlined.

\section{TEM-Wire Horn Antenna}

In Figure 1, a wire-type feed horn-fed parabolic reflector antenna is shown in Figure 1. The input signal is applied to the feed horn whose end points are attached to the surface of the parabola supporting itself. The horn is electrically 


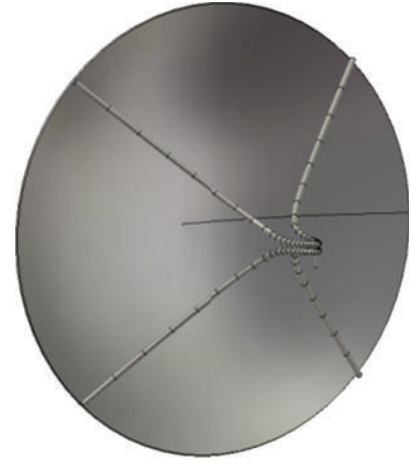

(a) Perspective

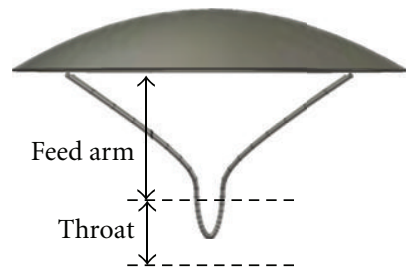

(b) Top view

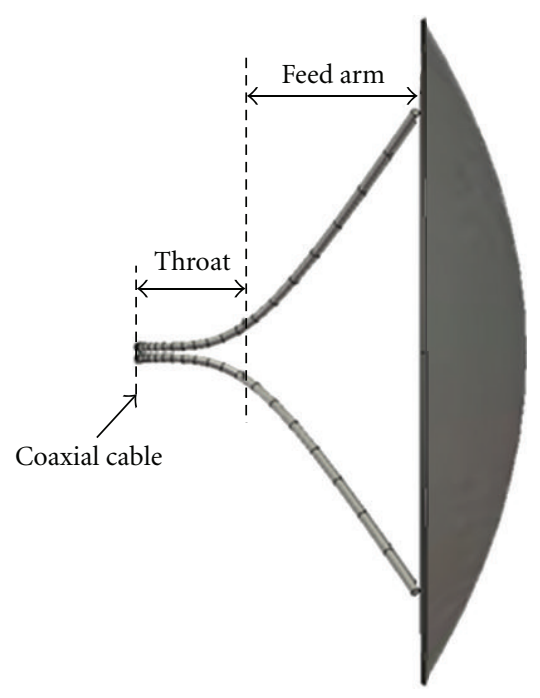

(c) left view

FIGURE 1: Wire-type feed horn fed parabolic reflector.

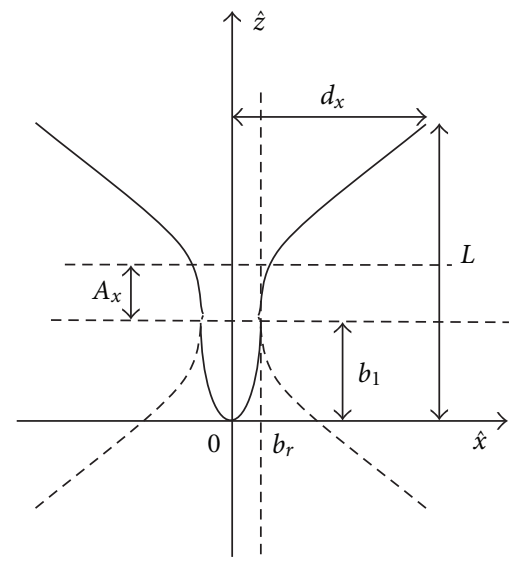

(a) $x z$-cut of the TEM-wire horn

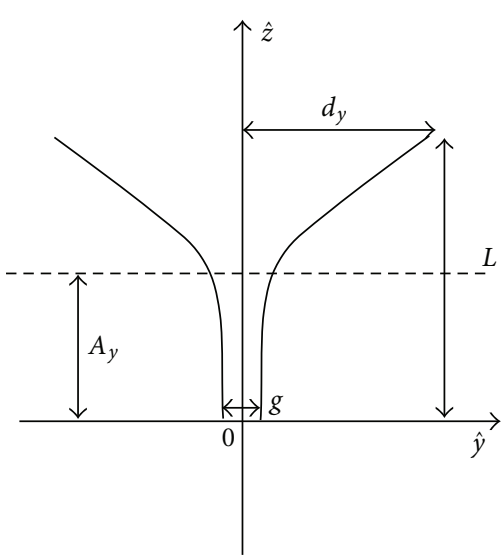

(b) $y z$-cut of the TEM-wire

Figure 2: Structure of the TEM-wire horn.

isolated from the parabolic reflector. In order to make the antenna light-weighted, the feed horn is made of copper wires or pipes whose diameter is $r_{\text {horn }}$.

The TEM-wire horn is obtained from a combination of two regions, which are the throat region and the tapered feed arm. Throat region is needed to transform the input impedance of a TEM-wire horn to the characteristic impedance of a coaxial cable. It also acts as a balun. Figure 2 shows the structure of a TEM-wire horn. The origin of the $z$-coordinate is at point where the coaxial cable is connected. The $z$-axis is in the direction of the parabolic surface. The tapered feed arm plays the role of guiding the incident wave to the parabolic surface with tolerable reflection. To smoothly guide the incident wave toward the surface of a parabolic reflector, a set of equations of parametric curves are devised for $x(z)$ and $y(z)$ as in (1). Control parameters for $x(z)$ are $A_{x}, b_{l}, b_{r}, n_{x}$, and $N_{x}$. Parameters for $y(z)$ are $A_{y}, n_{y}$, and $N_{y}$. Parameters $b_{r}$ and $b_{l}$ determine the shape of throat region. The width of throat is equal to $2 b_{r}$. The length of the throat is $b_{l}$. Parameters $N_{x}$ and $N_{y}$ determine asymptotic behaviors of feed arms when $z$ becomes large. As the $z$ coordinate increases, it becomes similar to a polynomial function of order $N_{x}$ or $N_{y}$. Parameters $n_{x}$ and $n_{y}$ affect the curve shape when $z$ is small. Parameters $A_{x}$ and $A_{y}$ determine the length of transition regions along $x$ and $y$ axes. In (1), $d_{x}$ and $d_{y}$ are distances along $x$ and $y$ axes from the feed point to 


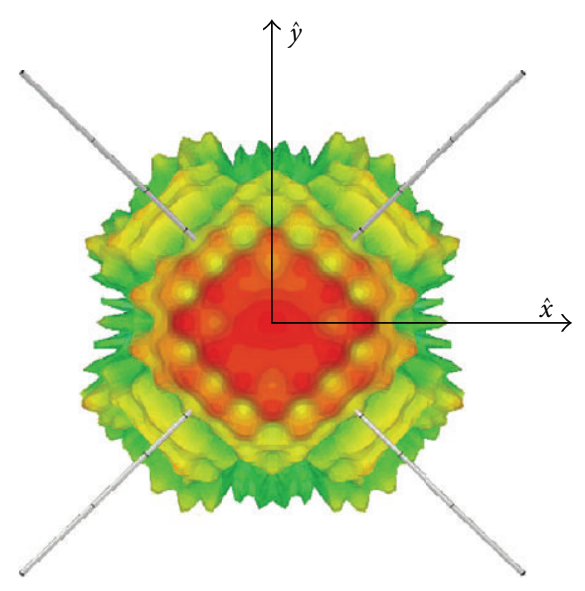

(a) Narrow throat at $3 \mathrm{GHz}$

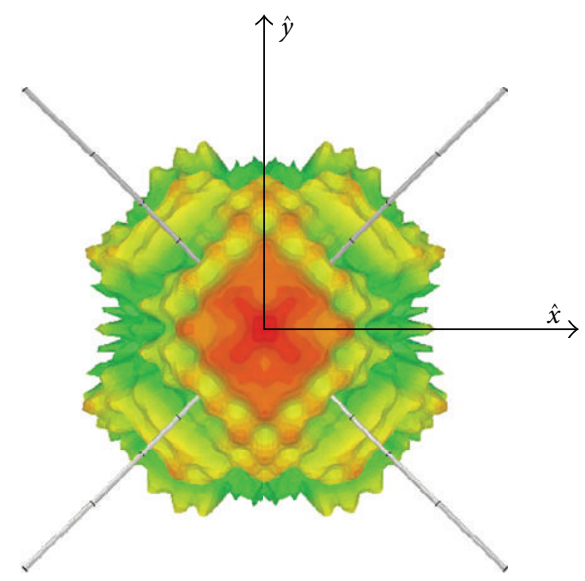

(c) Wide throat at $3 \mathrm{GHz}$

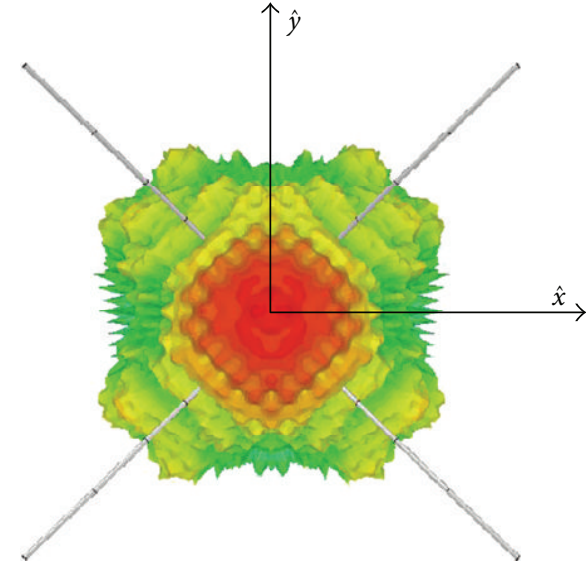

(b) Narrow throat at $5 \mathrm{GHz}$

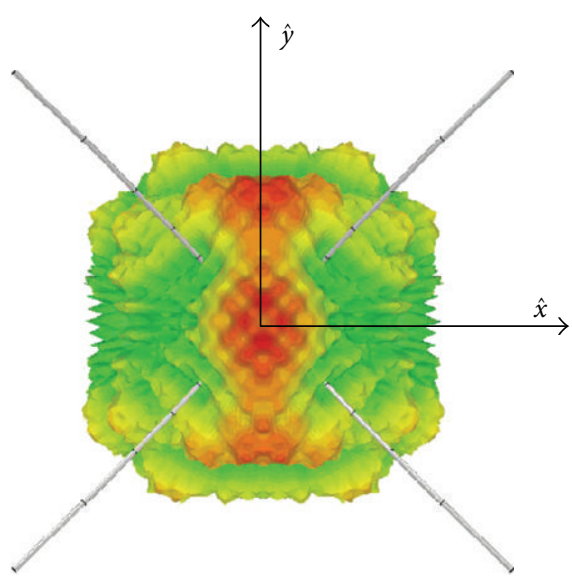

(d) Wide throat at $5 \mathrm{GHz}$

FIGURE 3: Comparison of three-dimensional radiation patterns of the two TEM-wire horns. With a wide throat region, the symmetry along both of the $x$ - and $y$-axes is broken at higher frequency.

the ends of feed arms. The vertical separation between feed wires is denoted by $g$. Those parameters are used to change the shape of a TEM-wire fed horn antenna

$$
\begin{gathered}
x(z)= \begin{cases}b_{r}+\left(d_{x}-b_{r}\right) \frac{\sqrt[n_{x}]{\left[\left(z-b_{l}\right) / A_{x}\right]^{n_{x}+N_{x}}+1}-1}{\sqrt[n_{x}]{\left[\left(L-b_{l}\right) / A_{x}\right]^{n_{x}+N_{x}}+1}-1}, & \text { for } z \geq b_{l}, \\
b_{r} \sqrt[n_{x}]{1-\left(1-\frac{z}{b_{l}}\right)^{n_{x}+N_{x}}}, & \text { for } z \leq b_{l},\end{cases} \\
y(z)=0.5 g+\left(d_{y}-0.5 g\right) \frac{\sqrt[n_{y}]{\left(z / A_{y}\right)^{n_{y}+N_{y}}+1}-1}{\sqrt[n_{y}]{\left(L / A_{y}\right)^{n_{y}+N_{y}}+1}-1} .
\end{gathered}
$$

The input port of the feed horn converts an unbalanced input signal of the coaxial cable into a balanced mode of the TEM-wire horn antenna, whose shape is effectively a tapered two-wire transmission line. The vertical separation between the copper pipes, which form the feed horn, can be determined in such a way that the parallel combination of the characteristic impedances of two-wire transmission lines should be equal to that of coaxial cable when the width of the throat region is assumed to be large

$$
\begin{aligned}
Z_{\text {coax }} & =\frac{1}{2 \pi} \frac{\eta_{0}}{\sqrt{\varepsilon_{r, \text { coax }}}} \ln \frac{r_{\text {out }}}{r_{\text {in }}}=\frac{1}{2} Z_{\text {horn }} \\
& =\frac{1}{2 \pi} \eta_{0} \cosh ^{-1}\left(\frac{d}{2 r_{\text {horn }}}\right),
\end{aligned}
$$

where $d$ is the separation between the centers of copper pipes and $r_{\text {horn }}, r_{\text {out }}$, and $r_{\text {in }}$ are the radii of the copper pipe, outer, and inner conductor of the coaxial cable, respectively

$$
d=2 r_{\text {horn }} \cosh \left(\frac{2 \pi Z_{\text {coax }}}{\eta_{0}}\right)
$$




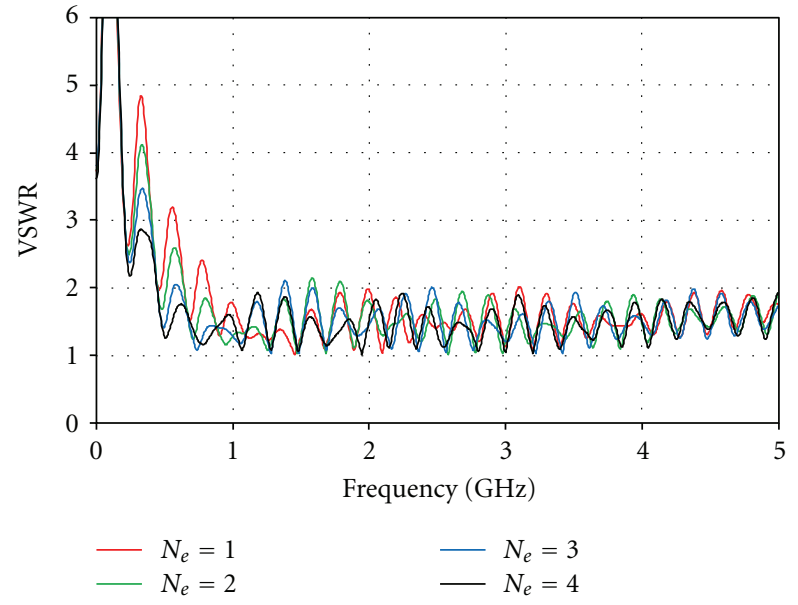

FIgURE 4: Comparison of return losses of the TEM-wire parabola with $N_{e}=1,2,3$, and 4 .

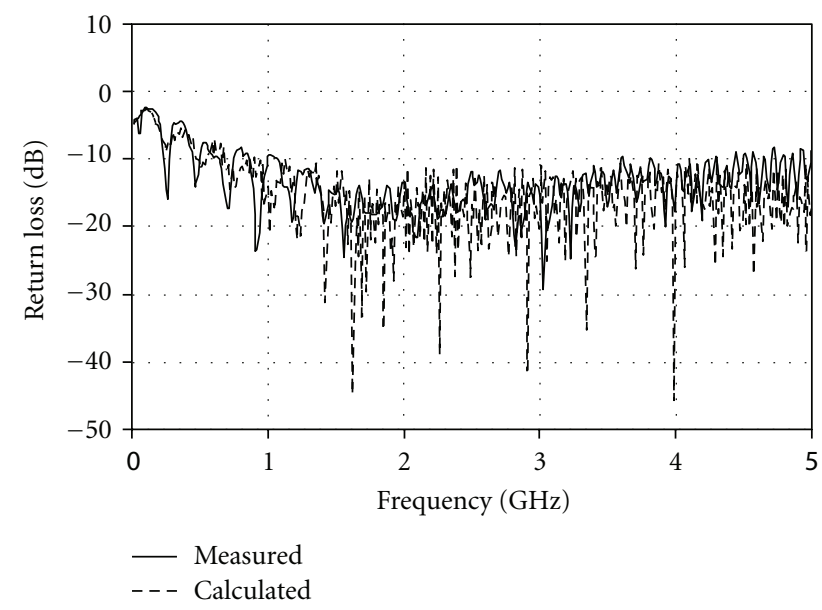

Figure 5: Comparison of measured results and those of a simulation for $N_{e}=4$ with a narrow throat. Simulation results show similar tendency to measured ones.

TABLE 1: Parameter values for TEM-wire horn curves with a narrow throat. Values in the parentheses are for a wide throat. (unit: $\mathrm{mm}$ ). The radius of wires is set to $5 \mathrm{~mm}$.

\begin{tabular}{llllcc}
\hline$d_{x}$ & 424.26 & $n_{x}$ & 2 & $A_{x}$ & 50 \\
$d_{y}$ & 424.26 & $n_{y}$ & 2 & $A_{y}$ & $50(100)$ \\
$b_{r}$ & $10(30)$ & $N_{x}$ & 4 & $g$ & 4 \\
$b_{l}$ & $30(50)$ & $N_{y}$ & 4 & $L$ & 490 \\
\hline
\end{tabular}

However, (3) does not hold when the width of the throat is small, in which case two arms of the TEM-horn can be considered as coupled TEM transmission lines. Due to the symmetry about $y z$-plane, even mode of the coupled lines is excited and the input impedance of the TEM horn becomes larger than the value obtained using (2).

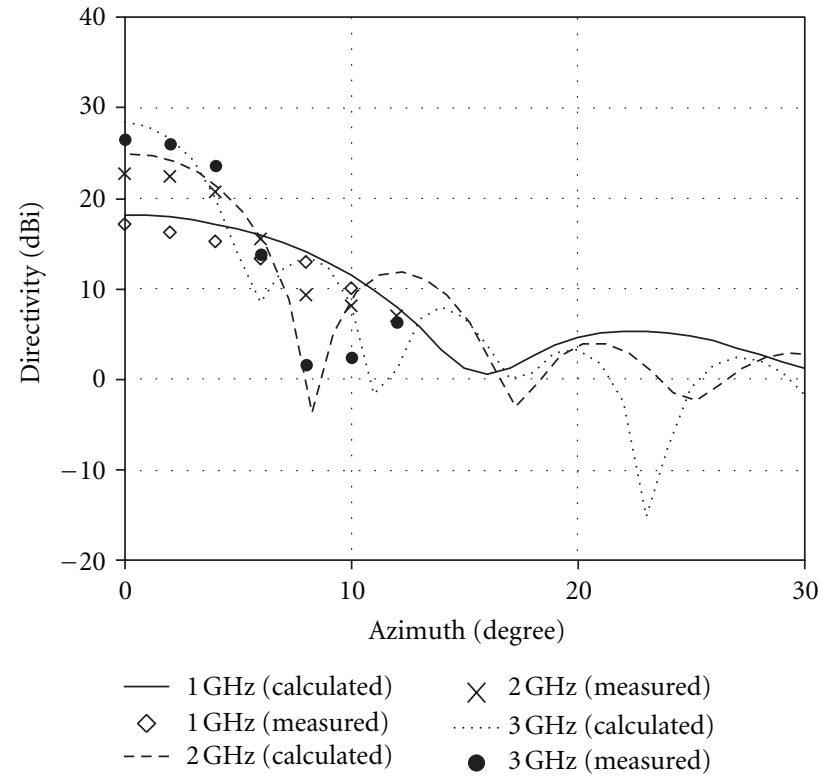

FIGURE 6: Comparison of measured radiation patterns and those of a simulation for $N_{x}=N_{y}=N_{e}=1$ with a narrow throat region. Simulation results show good agreement. Zero degree corresponds to the direction of the main beam.

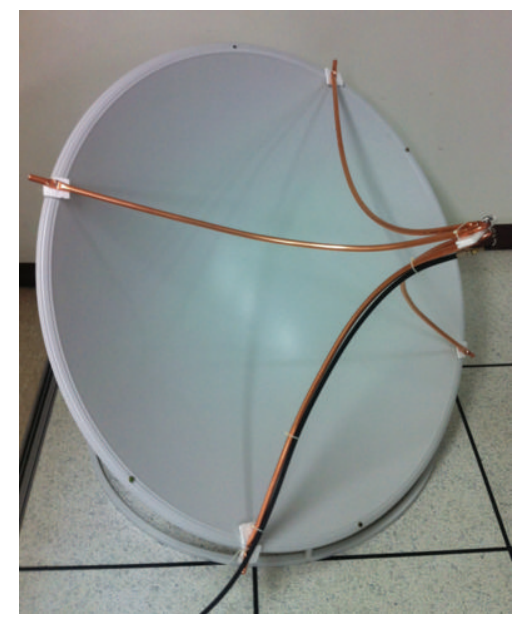

FIGURE 7: A fabricated TEM-wire fed parabola. Four ends of the TEM-wire horn are terminated by $150 \Omega$ resistors to minimize return losses below $1 \mathrm{GHz}$.

Once the input signal goes through the coax-to-two wire transition, it enters into a tapered section of the feed horn. Due to the increasing separation between copper pipes, the reflections are generated continuously on the way to the parabolic surface. To suppress the reflection under a tolerable level, the rate of increasing separation between two copper wires should be controlled to a predetermined level. To determine the rate of increasing separation, reflection coefficient formula for tapered lines [13] can be used. The 


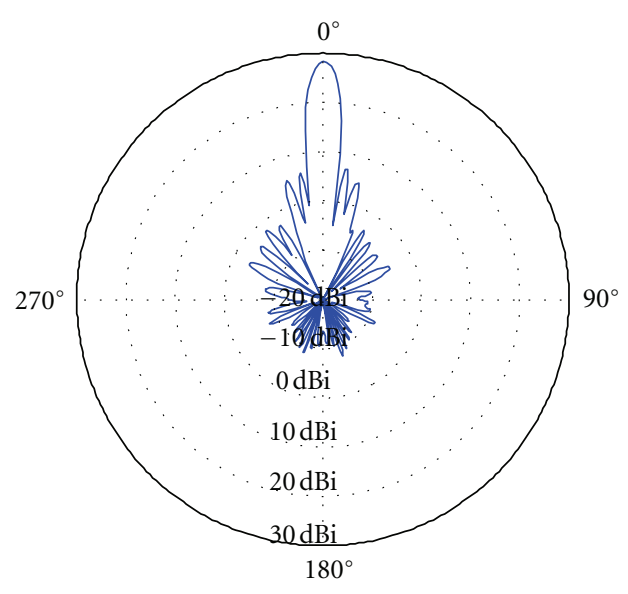

(a) $N_{e}=1$, peak value $28.3 \mathrm{dBi}$

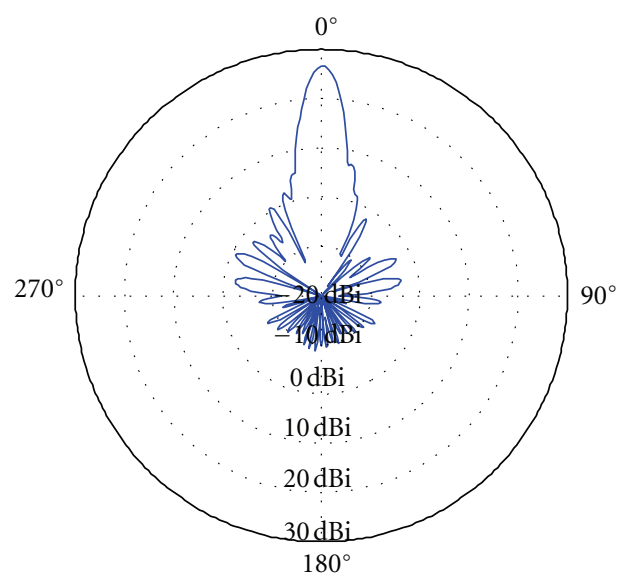

(c) $N_{e}=3$, peak value $26.6 \mathrm{dBi}$

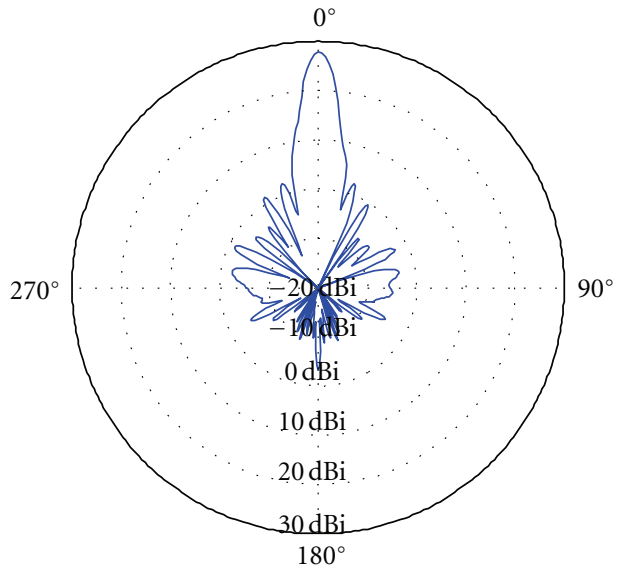

(b) $N_{e}=2$, peak value $27.9 \mathrm{dBi}$

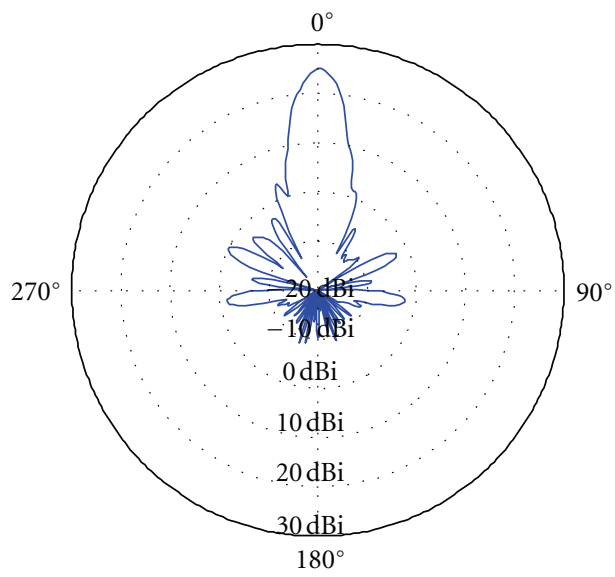

(d) $N_{e}=4$, peak value $25 \mathrm{dBi}$

Figure 8: Comparison of radiation patterns with different $N_{e}$ values at $3 \mathrm{GHz}$. Peak directivities decrease with larger $N_{e}$ and main lobe widths increase. The parameter $L$ in (1) is set to be $350 \mathrm{~mm}$. The other values except $N_{x}=N_{y}=N_{e}$ remain unchanged.

shape of the TEM-wire horns of (1) becomes asymptotes to polynomial curves of the order of $N_{x}$ or $N_{y}$ along $x$ or $y$-axis as the incident wave goes away from the throat region. The orders of polynomials are closely related to return losses. The higher is the order of the polynomial, the smaller the reflection occurs. At the same time, to make a compact antenna, it is desirable that the length of the throat be minimized. On the other hand, the length should be long enough to suppress the reflections at the input port. With the parametric curve of (1), requirements on the size of throat region as well as the asymptotic shape of the feed horn can be met. Return loss variations according to the order of polynomial will be mentioned again in Section 3 where the TEM-wire horn is attached to a parabolic reflector.

Radiation patterns of the TEM-wire feed horn are important in that they determine far field patterns of the reflector antenna. To accomplish high directivity when applied to a circular symmetric parabolic reflector, the incident wave should have a rotationally symmetric radiation pattern. The presented TEM-wire structure in Figure 1 has asymptotical symmetry about $\mathrm{E}$ and $\mathrm{H}$ plane in the feed arm region. The feed arms are bent so that they make right angle asymptotically. But the TEM-wire structure has nonsymmetric shape in the throat region. When the frequency of the applied input signal is low enough, the currents on the wire structure are formed mainly along the feed arm region whose asymptotically symmetric shape results in symmetric radiation pattern. As the frequency increases, most of the current is concentrated near the throat region where asymmetric structure is located. The asymmetric current induces asymmetry of the radiation field, which becomes the incident wave on the parabolic reflector. Figure 3 shows the $3 \mathrm{D}$ radiation pattern of the TEM-wire horn detached from a parabolic reflector. Shape parameters for (1) are listed in Table 1.

At low frequency, radiation patterns are invariant after rotating by an angle of 90 degrees. As the frequency increases, the radiation patterns become distinct. With narrow throat, 


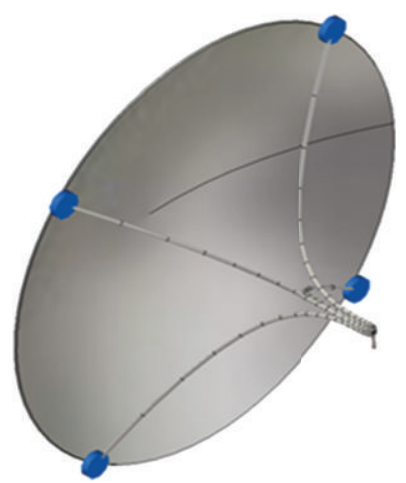

(a) Narrow throat

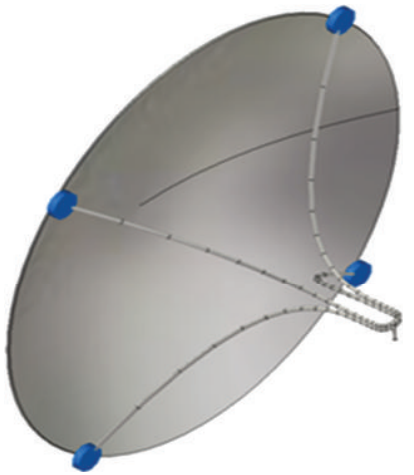

(b) Wide throat

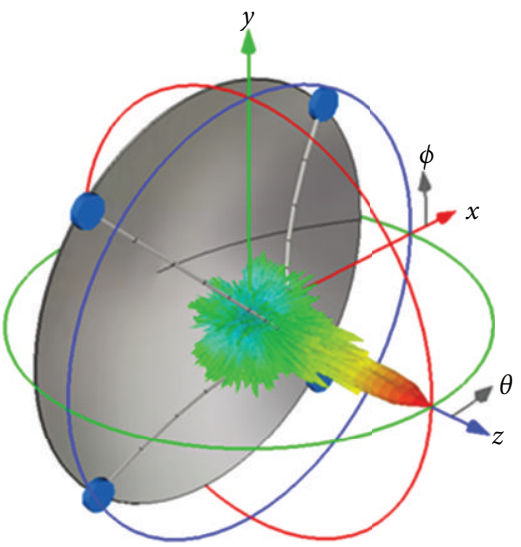

(c) Radiation pattern at $5 \mathrm{GHz}$ (narrow throat)

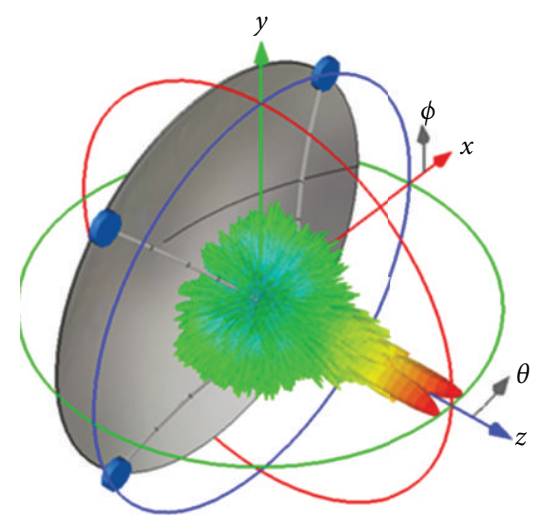

(d) Radiation pattern at $5 \mathrm{GHz}$ (wide throat)

FIGURE 9: Comparison of three-dimensional radiation patterns of the two TEM-wire horns attached to parabolic reflectors. With a wide throat region, the main beam is split into two peaks due to asymmetry of the pattern of the TEM-wire horn.

the symmetry of the radiation pattern is kept. With wide throat, the radiation pattern loses invariance after rotation. When this TEM-wire horn is attached to a parabolic reflector, the main lobe of the parabolic antenna gets split to have two peaks in radiation pattern due to the asymmetry of the incident wave. This phenomenon lowers directivity and limits operating frequency of the TEM-wire feed parabolic reflector antenna. With a narrow throat region, the frequency range in which the main beam has single peak becomes wider.

\section{TEM-Wire Fed Parabola}

The incident wave due to the feed horn propagates toward the parabolic reflector through the feed arm region. The shape of feed arms affects return losses and radiation property. Figure 4 shows VSWR's for TEM-wire fed parabolas with $N_{x}=N_{y}=N_{e}=1,2,3$, and 4, with the other parameters equal to those of Table 1. As the order of parametric curve increases, the frequency range where VSWR is lower than 2 increases. Thanks to the parabolic reflector, which transforms incident spherical waves into nearly plane waves, impedance matching is achieved over a very wide frequency range. It can be observed that the lower frequency bounds are influenced by $N_{e}$. Figure 5 shows the measured return losses and simulated ones, which shows similar results confirming validity of a simulator used for this work. As shown in the figure, impedance matching is done for the frequency band higher than $500 \mathrm{MHz}$. The upper limit is determined by behaviors of radiation patterns. Figure 6 shows the measured and simulated radiation patterns to confirm the validity of the simulator. With $N_{x}=N_{y}=N_{e}=1$, peak directivities at each frequency increase. Figure 7 shows a fabricated TEM-wire fed parabolic antenna with the parabola diameter equal to $1200 \mathrm{~mm}$ and its depth equal to $185 \mathrm{~mm}$. The $f / D$ ratio of the chosen parabola is 0.38 .

The order of parametric curve also affects radiation pattern. The incident wave from the TEM-wire horn is reflected from the parabola. Then, the reflected waves propagate parallel to the axis of the parabola with the equiphase plane perpendicular to the axis. This is the case only if the incident wave can be considered to emanate from the focal point of the parabolic surface. In other words, the phase center of the incident wave should coincide with the focus of the parabola. However, the location of the phase center depends on the shape of the TEM-wire horn. Figure 8 compares azimuthal patterns of the TEM-wire parabolic antennas with different $N_{e}$ values at $3 \mathrm{GHz}$. For $N_{e}$ equal to unity, the phase center of the TEM-wire horn can be found from the intersection of asymptotes of feed arm wires. For $N_{e}$ larger than one, phase centers cannot be determined simply. From Figure 8, the phase centers of TEM-wire horns seem to move closer to the surface of a parabolic reflector from the focus of the parabolic reflector with increasing $N_{e}$.

Figures 9 and 10 show the radiation patterns of the two TEM-wire feed horns with different throat widths attached to the same parabolic reflector as mentioned in Section 2. With a narrow throat width, radiation patterns have one peak and higher directivity at $5 \mathrm{GHz}$. With a wide throat width, the main lobe is split into two peaks at $5 \mathrm{GHz}$, thus has lower directivity than a narrow throat width. At this frequency, the asymmetric shape of the throat region of the TEM-wire horn limits increase of directivity.

Figure 11 compares directivities of TEM-wire fed parabolas with different throat widths. As mentioned before, the parabola with wide throat has its main lobe split so that its increase of directivities is limited. With a wide throat 


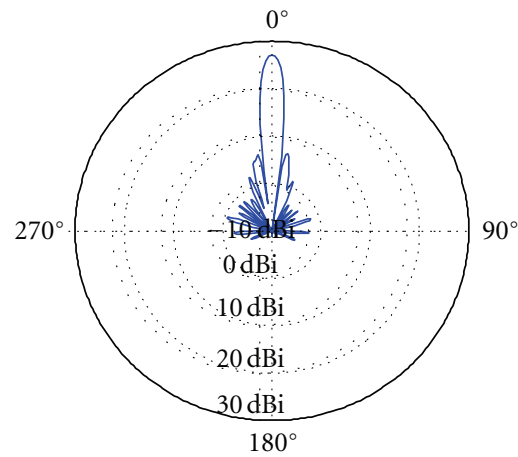

(a) Narrow throat, $3 \mathrm{GHz}$

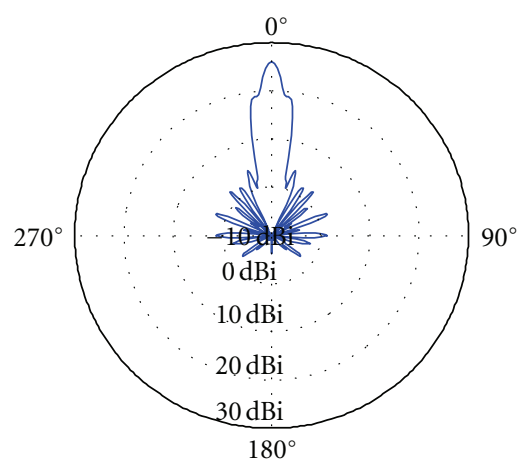

(d) Wide throat, $3 \mathrm{GHz}$

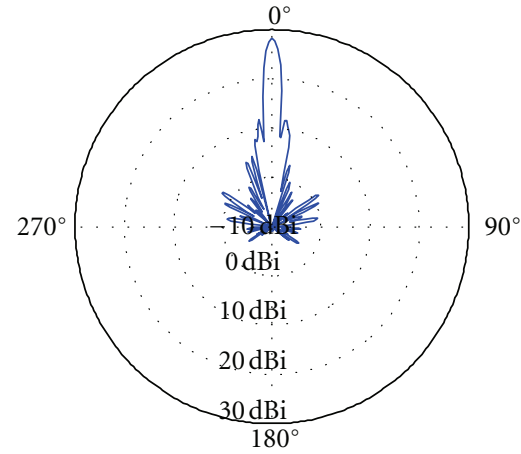

(b) Narrow throat, $4 \mathrm{GHz}$

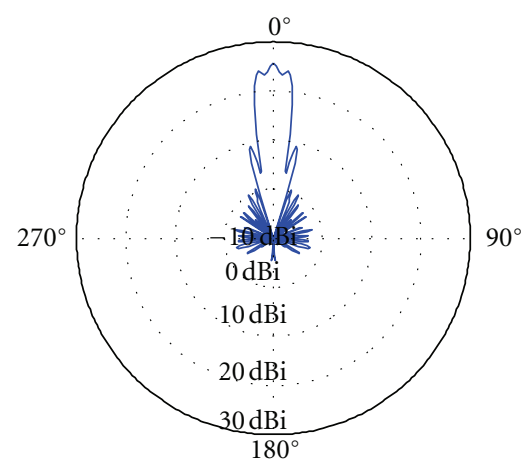

(e) Wide throat, $4 \mathrm{GHz}$

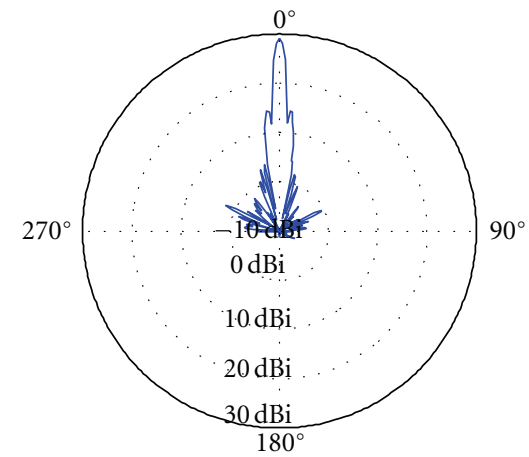

(c) Narrow throat, $5 \mathrm{GHz}$

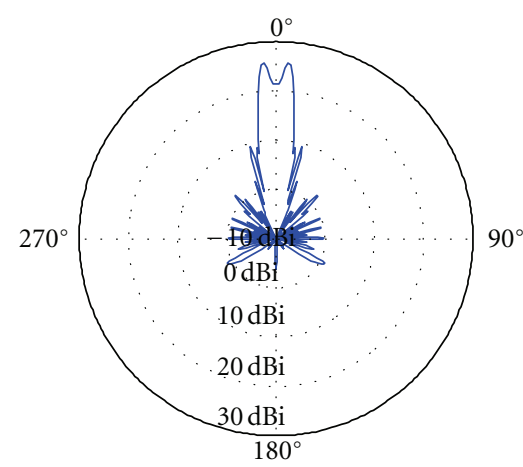

(f) Wide throat, $5 \mathrm{GHz}$

FIGURE 10: H-plane radiation patterns of the two TEM-wire fed parabolic antennas with a narrow throat-(a), (b), and (c). Those of a wide throat (d), (e), and (f).

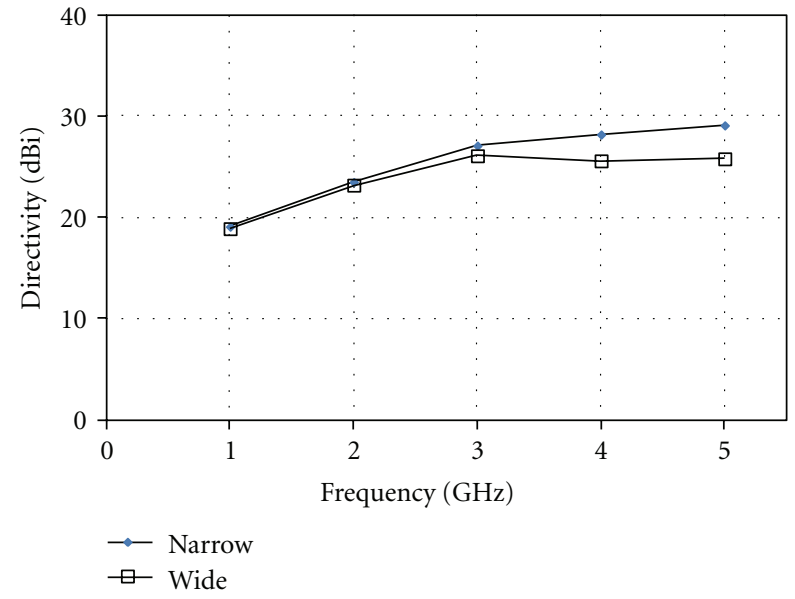

Figure 11: Comparison of directivities of the two TEM-wire parabolas with narrow and wide throat regions. With a wide throat, directivities do not increase anymore above $3 \mathrm{GHz}$.

region, the phase centers of the incident fields along the left and right arms fall apart from the focus of the parabola, of which state is similar to the case of [14].

Figure 12 shows efficiencies of the TEM-wire fed parabola. As the frequency increases, total efficiency approaches unity until $4 \mathrm{GHz}$. The high frequency performance is influenced by a bent region near the coaxial feed point, which

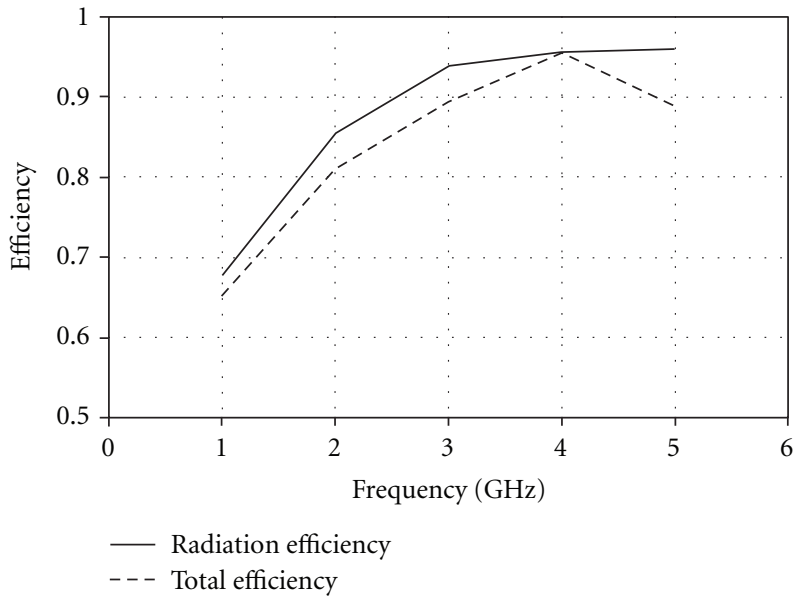

Figure 12: Efficiencies of a narrow throat antenna $\left(N_{e}=4\right)$ approach unity as the frequency increases.

has nonsymmetric shape. With a wide throat feed arm, the frequency of peak total efficiency becomes lower.

Figure 13 shows the time domain performance of the antenna. The copolar component of electric fields has the shape of a differentiation of the incident voltage waveform. As shown in Figure 13(c), high frequency responses are limited by the width of throat region. With a narrow throat, 


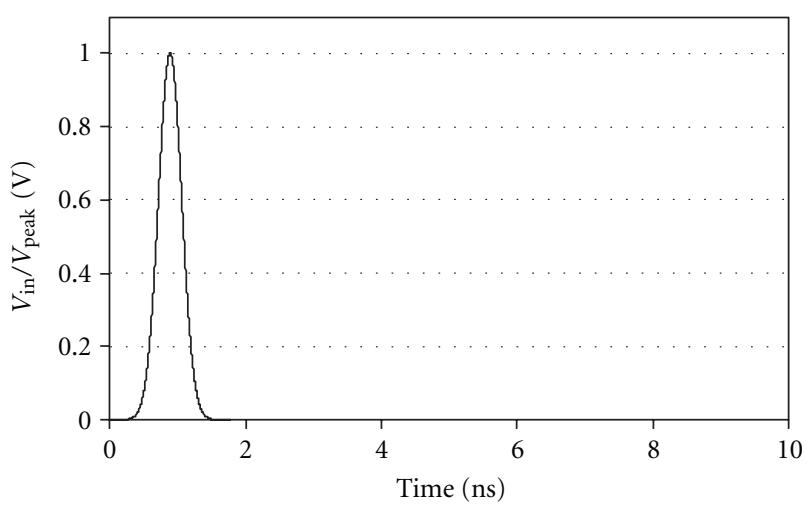

(a) Incident voltage waveform

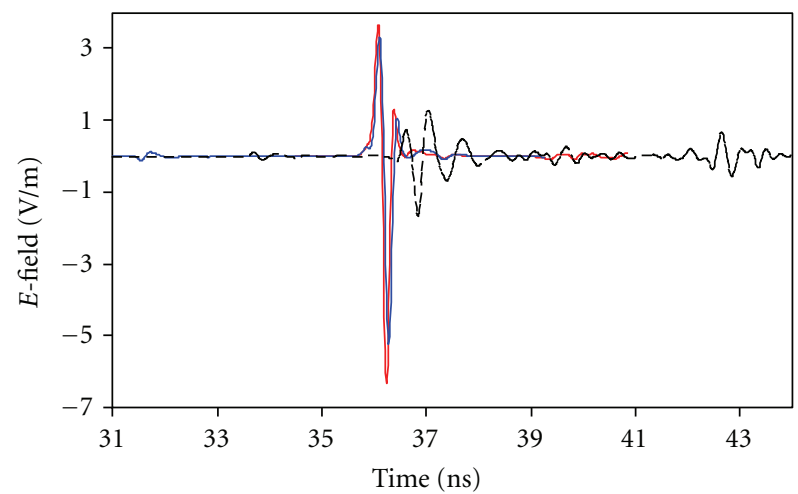

_ Narrow TEM horn
—_ Wide TEM horn
-- Rectangular horn feed

(b) $E_{y}$ component

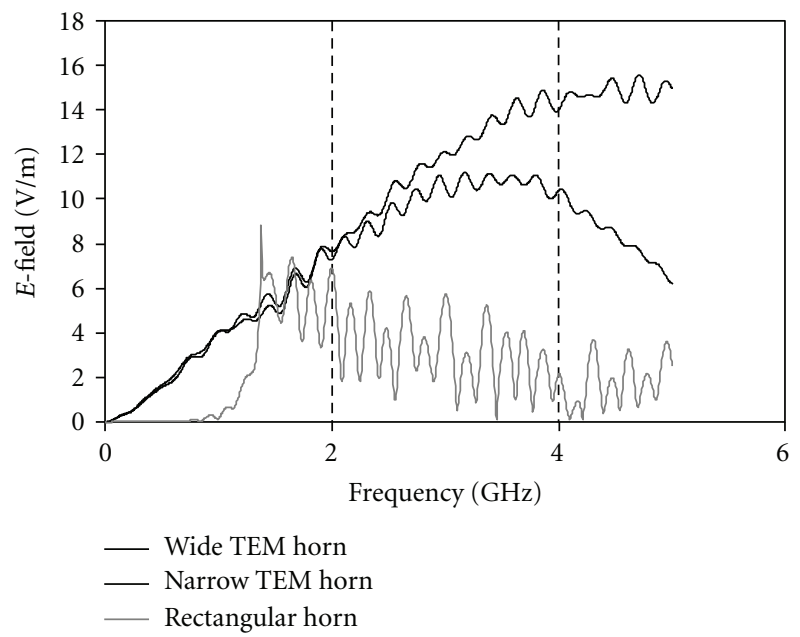

(c) Fourier transform of $E_{y}$ components

Figure 13: Calculated E-field obtained from a commercial simulator at a distance of $10 \mathrm{~m}$ from the center of the parabolic antenna. The incident voltage peak is $7.07 \mathrm{~V}$.

magnitudes of voltage peaks are larger than those of a wide throat. Electric fields of a parabola with a conventional standard rectangular feed horn are also drawn in the figure to compare the performance of TEM horn fed parabolic antenna, which preserves a short pulse width. The aperture size of the feed horn used is $313 \times 232 \mathrm{~mm}$. Compared with the TEM feed horn, the rectangular feed horn does not maintain short pulse width of the incident Gaussian pulse.

\section{Conclusion}

In this paper, a parametric curve equation for ultra-wide band antennas with very high directivity is proposed and the properties like return losses, radiation patterns, and time domain responses are investigated. Although the structure can be built at low cost by bending copper wires or pipes, asymmetric shape near the throat region forces the main lobes to be split, which limit increase of directivity with frequency. By careful design considerations, the frequency range of the ultra-wide band antenna can be extended.

\section{References}

[1] C. E. Baum and E. G. Farr, "Impulse radiating antennas," in Ultra Wideband/Short Pulse Electromagnetics, H. L. Bertoni, C. E. Baum, and L. B. Felsen, Eds., pp. 139-147, Plenum Press, New York, NY, USA, 1993.

[2] Z. Chen and Y. P. Zhang, "Effects of antennas and propagation channels on synchronization performance of a pulse-based ultra-wideband radio system," Progress in Electromagnetics Research, vol. 115, pp. 95-112, 2011.

[3] R. K. Amineh, A. Trehan, and N. K. Nikolova, "TEM horn antenna for ultra-wide band microwave breast imaging," Progress In Electromagnetics Research B, no. 13, pp. 59-74, 2009.

[4] P. Knott and A. Bell, "Coaxially-fed tapered slot antenna," Electronics Letters, vol. 37, no. 18, pp. 1103-1104, 2001. 
[5] R. H. DuHamel, M. E. Armstrong, J. J. Campbell, W. R. Jones, and W. F. Pedler, "Frequency independent conical feeds for lens and reflectors," in Proceedings of IEEE Antennas and Propagation Society International Symposium, vol. 6, pp. 414418, 1968.

[6] H. E. King and J. L. Wong, "Measured RF characteristics of a TEM-wire fed reflector," in Proceedings of the Antennas and Propagation Symposium Digest, vol. 1, pp. 266-269, May 1990.

[7] C. E. Baum, E.G. Farr, and D. V. Giri, "Review of impulse radiating antennas," in Review of Radio Science, W. R. Stone, Ed., pp. 403-439, Oxford University Press, Oxford, UK, 1999.

[8] L. H. Bowen, E. G. Farr, and W. D. Prather, "A high-voltage cable-fed impulse radiating antenna," Sensor and Simulation Notes 507, 2005.

[9] J. S. Tyo, "Optimization of the TEM feed structure for fourarm reflector impulse radiating antennas," IEEE Transactions on Antennas and Propagation, vol. 49, no. 4, pp. 607-614, 2001.

[10] M. Manteghi and Y. Rahmat-Samii, "A novel Vivaldi fed reflector impulse radiating antenna (IRA)," in Proceedings of IEEE Antennas and Propagation Society International Symposium and USNC/URSI Meeting, vol. 1A, pp. 549-552, July 2005.

[11] M. Manteghi and Y. Rahmat-Samii, "On the characterization of a reflector impulse radiating antenna (IRA): full-wave analysis and measured results," IEEE Transactions on Antennas and Propagation, vol. 54, no. 3, pp. 812-822, 2006.

[12] P. Zhang, Y. D. Yao, E. Fajanda, H. Moore, and H. Singh, "Impacts of different arm structures of reflector IRA: wire, coplanar plate, and bi-conical arms," in Proceedings of IEEE Sarnoff Symposium (SARNOFF '09), pp. 1-5, April 2009.

[13] D. M. Pozar, Microwave Engineering, John Wiley \& Sons, 2nd edition, 1998.

[14] C. Guo, A. X. Zhang, H. Wu, Y. S. Jiang, and W. B. Wang, "A high-power reflector impulse antenna with dual-TEM source," International Journal of Infrared and Millimeter Waves, vol. 29, no. 9, pp. 832-838, 2008. 

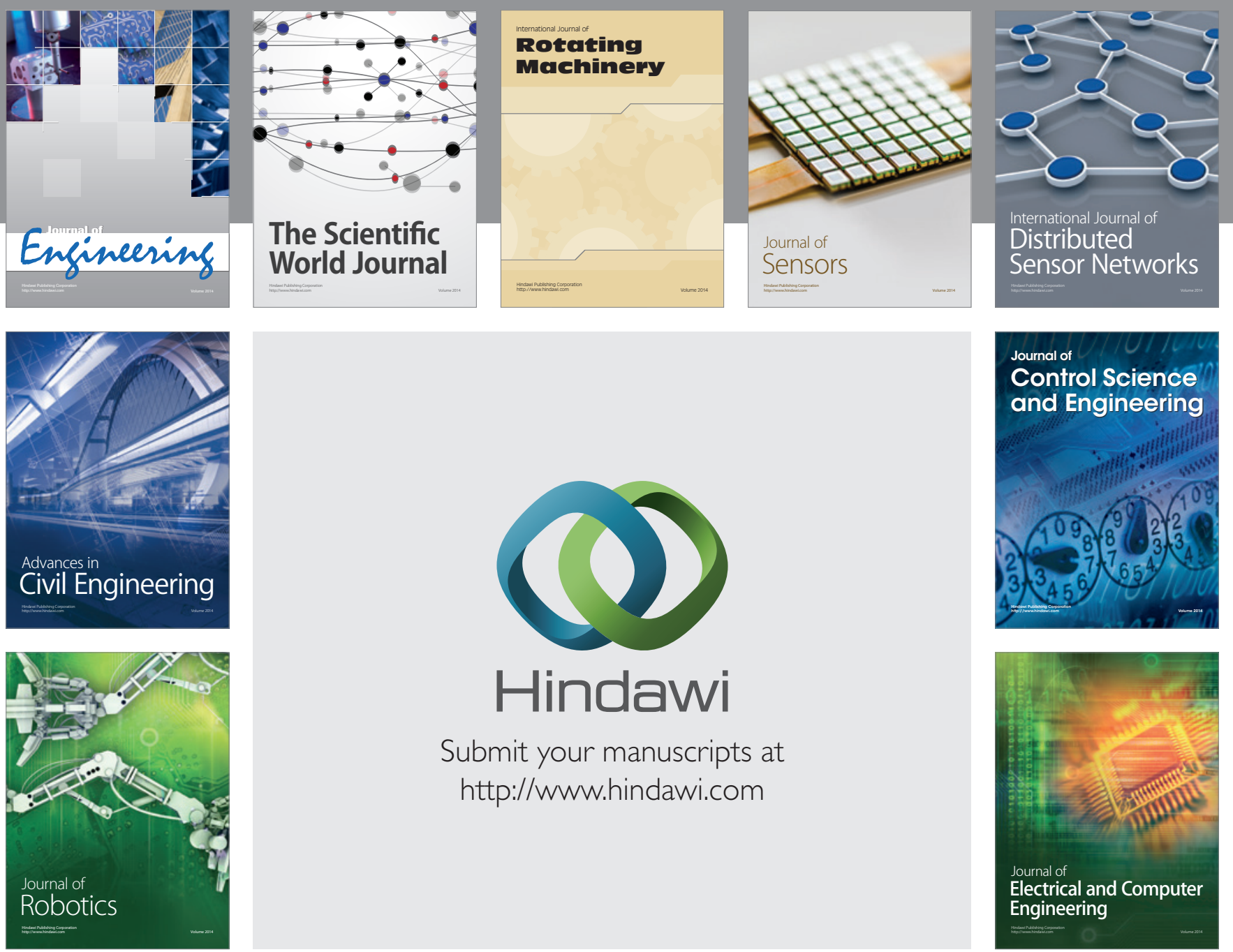

Submit your manuscripts at

http://www.hindawi.com
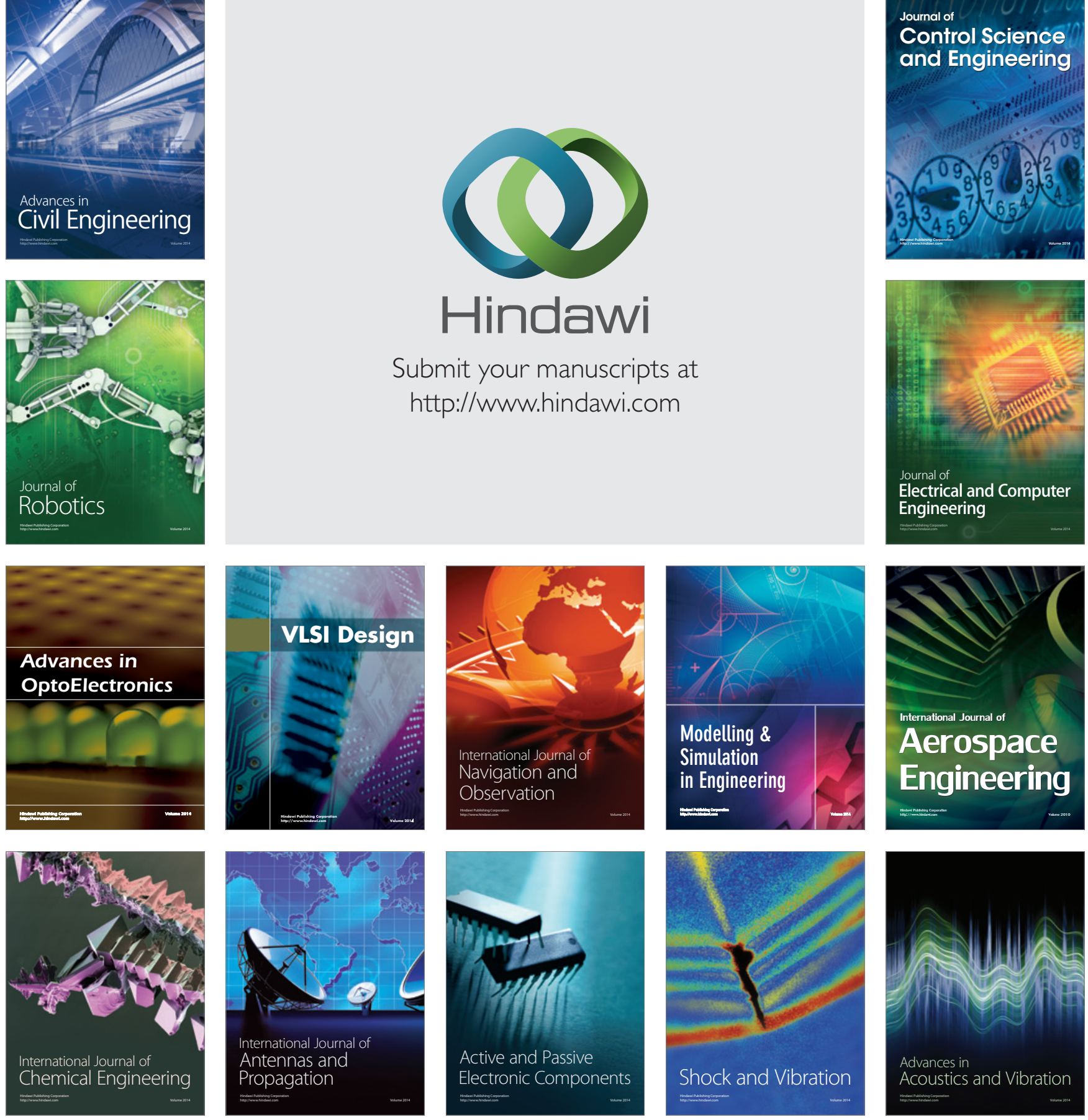